\title{
Desquamative interstitial pneumonia: still orphan and not always benign
}

\author{
Vincent Cottin (1)
}

Affiliation: National Reference Centre for Rare Pulmonary Diseases, Louis Pradel Hospital, Hospices Civils de Lyon, UMR 754, Claude Bernard University Lyon 1, Lyon, France.

Correspondence: Vincent Cottin, Dept of Respiratory Medicine, National Coordinating Reference Center for Rare Pulmonary Diseases, Louis Pradel Hospital, Hospices Civils de Lyon, 28 avenue Doyen Lepine, 69677, Lyon, France. E-mail: vincent.cottinđachu-lyon.fr

@ERSpublications

In 2020, DIP is no longer idiopathic but remains orphan within the group of interstitial pneumonia. Disease course is not always benign. International collaboration is warranted to enable prospective studies. https://bit.ly/2YmR6B0

Cite this article as: Cottin V. Desquamative interstitial pneumonia: still orphan and not always benign. Eur Respir Rev 2020; 29: 200183 [https://doi.org/10.1183/16000617.0183-2020].

Desquamative interstitial pneumonia (DIP) was originally described by LieBow et al. [1] in 1965, and so named because of the observation of cells filling the alveolar spaces and the belief that this feature was due to desquamation of alveolar epithelial cells. It has since been recognised that the dominant histologic feature of DIP represents accumulation of intra-alveolar macrophages, and sometimes of giant cells [2]. Although the more accurate terminology of "alveolar macrophage pneumonia" has been proposed, the term DIP has persisted [3].

In the international classification of idiopathic interstitial pneumonias [3], DIP and respiratory bronchiolitis-interstitial lung disease (RB-ILD) belong to the group of smoking-related interstitial pneumonia, together with pulmonary Langerhans cell granulomatosis. Depending on the classification, combined pulmonary fibrosis and emphysema, smoking-related acute interstitial pneumonia, smoking-related interstitial fibrosis, rheumatoid arthritis-associated ILD, and idiopathic pulmonary fibrosis may also be considered as smoking-related ILDs $[4,5]$.

DIP and RB-ILD are part of a histologic spectrum of macrophage accumulation, with the distinction dependent on the extent and distribution of this process. Histologic distinction between DIP and RB-ILD is sometimes difficult, hence the terminology of smoking-induced ILD [6]. RB, a finding present in the lung of almost all smokers, is characterised by the deposition of tobacco pigmented-macrophages predominating in the respiratory bronchioles and peribronchiolar alveolar spaces [7]. RB-ILD is a form of interstitial pneumonia, where RB is associated with a chronic ILD predominating in peribronchial areas and resulting from extensive response to smoking, with pathologic, clinical, and radiologic features [8]. DIP is characterised by prominent, diffuse intra-alveolar accumulation of pigmented macrophages, hyperplasia of type II alveolar epithelial cells, and often diffuse alveolar septal thickening, with possible septal fibrosis and mild interstitial inflammation $[1,6,9]$. In addition to demonstrating a large number of pigmented macrophages, bronchoalveolar lavage may show an increased number of eosinophils [10], but whether eosinophils are associated with a different response to glucocorticoids or a different outcome is unclear.

Distinctions between DIP and RB-ILD also reflect in the pattern of disease on high-resolution computed tomography [11], with centrilobular nodules and ground-glass attenuation predominating in the upper

Provenance: Commissioned article, peer reviewed

Received: 7 June 2020 | Accepted: 9 June 2020

Copyright $\odot$ ERS 2020. This article is open access and distributed under the terms of the Creative Commons Attribution Non-Commercial Licence 4.0. 
lobes in RB-ILD, and ground-glass attenuation with lower lobe predominance (and rarely centrilobular nodules) in DIP (figure 1) [12-14]. In RB-ILD, pulmonary function tests can be normal or show a restrictive, obstructive or mixed pattern; however, in DIP a restrictive physiology with reduced diffusing capacity of the lung for carbon monoxide is more commonly observed [4].

In contrast with RB-ILD, which is invariably associated with tobacco smoking, a significant proportion of cases of DIP $(\sim 20 \%)$ were reported in nonsmokers. DIP has been related to a variety of causes other than

FIGURE 1 Representative highresolution computed tomography in a patient with biopsy-proven desquamative interstitial pneumonia showing ground-glass attenuation predominating in the medium and central zones of the lung. a) Coronal view. b) Transverse view at the level of inferior pulmonary veins. c) Transverse view in the lung bases. The patient quit smoking and disease course was stable and uneventful.
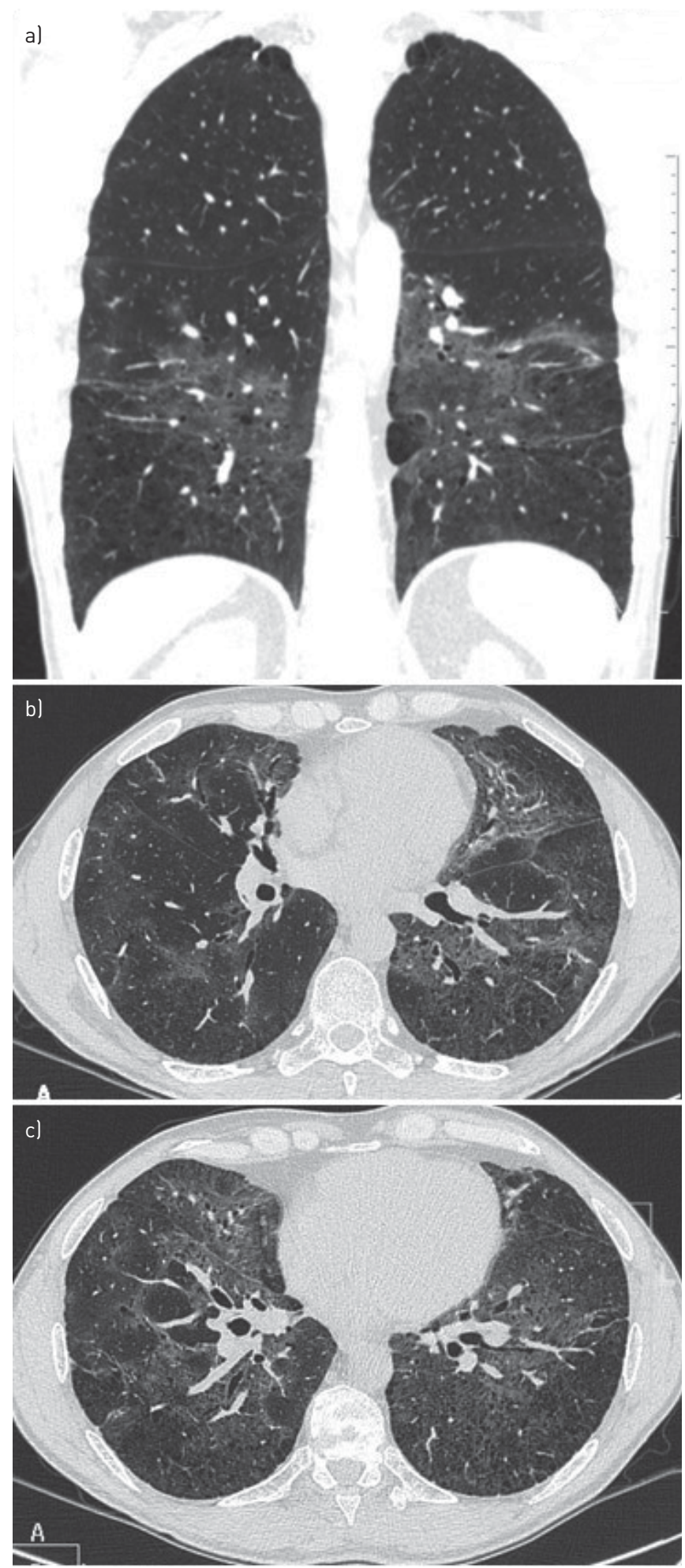
tobacco smoking, including connective tissue disease (rheumatoid arthritis, systemic sclerosis, rarely systemic erythematosus lupus), viral infections (hepatitis $\mathrm{C}$ virus, cytomegalovirus), occupational exposures to inorganic particles (solder fumes, flock-workers, diesel fumes, tungsten carbide), environmental exposures (mycotoxins, dust), medications (sirolimus, nitrofurantoin), and illicit drugs (cannabis) [4, 15]. DIP has also been reported in children, mostly in the setting of interstitial pneumonia of familial or genetic origin.

Despite the fact that DIP was first described 55 years ago, much remains to be learnt about this entity. Publications of series are scarce and spread over many years, limiting access to synthetic information. In the current issue of the European Respiratory Review, Hellemons et al. [16] performed a systematic literature search of DIP, and reviewed a total of 362 cases of DIP from 13 case series and 68 individual cases in adults published between 1965 and 2019. They reported dyspnoea and cough as the most common presenting symptoms, and presence of a restrictive physiology in $71 \%$ of cases with reduced diffusion capacity. Importantly, $19 \%$ of cases had never smoked, and a further $11 \%$ were former smokers, confirming that DIP can occur in the absence of active smoking. Although causality was difficult to establish, $22 \%$ of cases had occupational exposures. Imaging features were characterised by bilateral ground-glass opacities with lower lobe predominance in $92 \%$ of cases. While these results were not surprising, the systematic review provides us with a much more accurate overview than previously available from individual cases or small series.

Furthermore, Hellemons et al. [16] identified data regarding treatment received in a majority of cases. Removal of the suspected culprit and initiation of glucocorticoids were the most frequent treatments. However, data on outcome were relatively limited. Whether smoking cessation indeed affects the outcome of DIP has not been demonstrated. More than half of the patients with DIP who receive glucocorticoids seem to benefit from therapy [16-19] and have a relatively good prognosis [10, 16]; however, progression to chronic respiratory failure, need for transplantation or death were also reported [20, 21]. Among patients with a known smoking history, $70 \%$ of patients who had smoked had a stable course of disease or improved; in contrast, only $40 \%$ of those without a history of smoking improved or remained stable and the majority experienced disease worsening (especially in women) [16]. Imaging features at baseline were not predictive of outcome.

Such findings suggest that the pathogenesis of DIP may not be as uniform as the pathologic pattern suggests, and that the severity of disease may not be as benign as generally considered. DIP related to occupational exposures may not always be comparable to smoking-induced DIP, although the available data do not allow us to decipher between the respective roles of the nature of the culprit or of its

2005
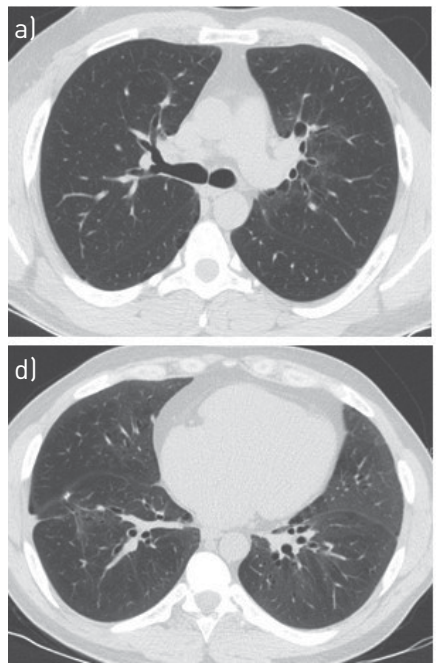

2013
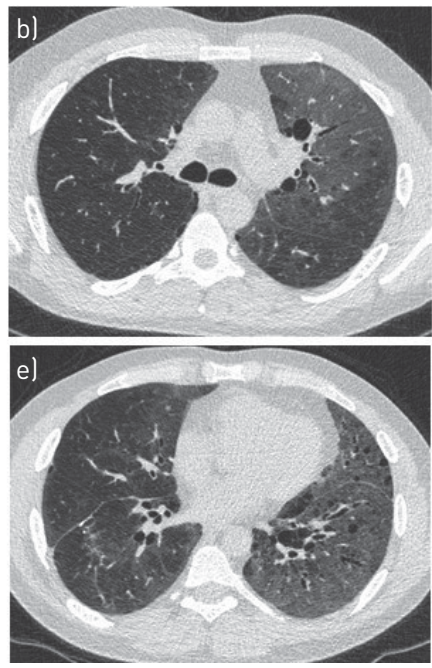

2017

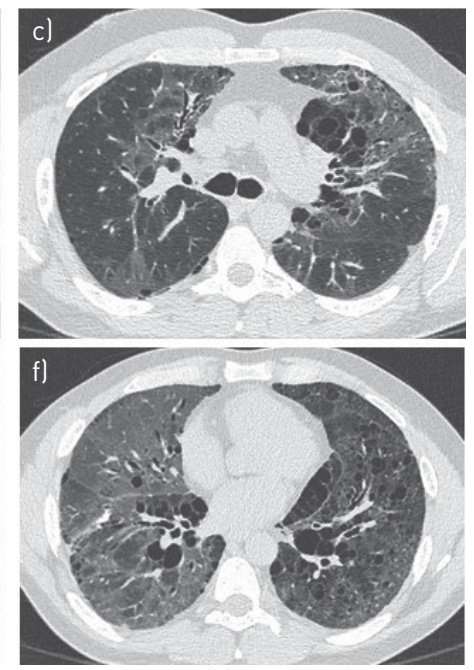

FIGURE 2 Representative high-resolution computed tomography in a patient with biopsy-proven desquamative interstitial pneumonia. The patient was an ex-smoker with occupational history of being a welder, aged 35 years at the time of the first high-resolution computed tomography scan. Follow-up computed tomography scans demonstrate worsening of imaging abnormalities, with increase in extent and density of ground-glass attenuation, and progression to multiple cystic lung disease. Transverse views at the level of the a-c) carina and d-f) inferior pulmonary veins in 2005 (a, d), 2013 (b, e) and 2017 (c, f). The patient developed chronic respiratory failure, underwent transplantation and pathology of explanted lung confirmed the diagnosis of desquamative interstitial pneumonia. 
persistence over the years. In other words, patients with DIP may be more likely to quit smoking than to definitely quit their job and any associated inhaled occupational exposure, and this difference might translate into a different outcome. The findings by Hellemons et al. [16] also underline that further studies are eagerly needed to improve our knowledge on the course of disease and best management of DIP. Over time, some cases of DIP remain stable on imaging while in others ground-glass attenuation progresses to a complex pattern of fibrosis and on occasion multiple cystic lesions distinct from honeycombing and from the usual interstitial pneumonia pattern (figure 2) [13, 22]. Very little information is available, however, regarding the effect of therapy other than glucocorticoids in DIP. In severe and progressive cases worsening despite smoking cessation and glucocorticoids, treatments that should be considered include immunosuppressive therapy [16], clarithromycine [23], antifibrotic therapy (especially nintedanib in chronic fibrosing forms of DIP) [24], and lung transplantation despite possible recurrence after transplantation [25].

In conclusion, as is often the case, the systematic review raises as many questions as it provides answers, and it emphasises that much remains to be known about DIP. Whether occupational exposure-related DIP is the same condition as smoking-related DIP is uncertain at this time. Although DIP is much less severe than idiopathic pulmonary fibrosis, not all cases of DIP have a benign disease course despite smoking cessation, in contrast with RB-ILD which generally has a benign course [26, 27]. Female sex and occupational exposure seem to be associated with a poor outcome. Cases at risk of fibrotic progression to chronic respiratory failure need to be identified early and warrant close monitoring and active management. In 2020, DIP is no longer idiopathic but remains orphan within the larger group of interstitial pneumonia. An "adoption" of DIP by the scientific community would need a kick start, with precise consensus diagnostic and classification criteria possibly obtained through a Delphi process, and international collaboration through prospective studies and international registries.

Conflict of interest: V. Cottin reports personal fees and non-financial support from Actelion and from Roche/ Promedior; grants, personal fees and non-financial support from Boehringer Ingelheim; and personal fees from Bayer/ MSD, Novartis, Sanofi, Celgene, Galapagos, Galecto, Shionogi, AstraZeneca and Fibrogen, outside the submitted work.

\section{References}

1 Liebow AA, Steer A, Billingsley JG. Desquamative interstitial pneumonia. Am J Med 1965; 39: 369-404.

2 Tubbs RR, Benjamin SP, Reich NE, et al. Desquamative interstitial pneumonitis. Cellular phase of fibrosing alveolitis. Chest 1977; 72: 159-165.

3 Travis WD, Costabel U, Hansell DM, et al. An official American Thoracic Society/European Respiratory Society statement: update of the international multidisciplinary classification of the idiopathic interstitial pneumonias. Am J Respir Crit Care Med 2013; 188: 733-748.

4 Kumar A, Cherian SV, Vassallo R, et al. Current concepts in pathogenesis, diagnosis, and management of smoking-related interstitial lung diseases. Chest 2018; 154: 394-408.

5 Margaritopoulos GA, Vasarmidi E, Jacob J, et al. Smoking and interstitial lung diseases. Eur Respir Rev 2015; 24: 428-435.

6 Yousem SA, Colby TV, Gaensler EA. Respiratory bronchiolitis-associated interstitial lung disease and its relationship to desquamative interstitial pneumonia. Mayo Clinic Proc 1989; 64: 1373-1380.

7 Niewoehner DE, Kleinerman J, Rice DB. Pathologic changes in the peripheral airways of young cigarette smoker's. N Engl J Med 1974; 291: 755-758.

8 Myers JL, Veal CF Jr, Shin MS, et al. Respiratory bronchiolitis causing interstitial lung disease. A clinicopathologic study of six cases. Am Rev Respir Dis 1987; 135: 880-884.

9 Craig PJ, Wells AU, Doffman S, et al. Desquamative interstitial pneumonia, respiratory bronchiolitis and their relationship to smoking. Histopathology 2004; 45: 275-282.

10 Kawabata Y, Takemura T, Hebisawa A, et al. Eosinophilia in bronchoalveolar lavage fluid and architectural destruction are features of desquamative interstitial pneumonia. Histopathology 2008; 52: 194-202.

11 Heyneman LE, Ward S, Lynch DA, et al. Respiratory bronchiolitis, respiratory bronchiolitis-associated interstitial lung disease, and desquamative interstitial pneumonia: different entities or part of the spectrum of the same disease process? AJR Am J Roentgenol 1999; 173: 1617-1622.

12 Hartman TE, Primack SL, Swensen SJ, et al. Desquamative interstitial pneumonia: thin-section CT findings in 22 patients. Radiology 1993; 187: 787-790.

13 Hartman TE, Primack SL, Kang EY, et al. Disease progression in usual interstitial pneumonia compared with desquamative interstitial pneumonia. Assessment with serial CT. Chest 1996; 110: 378-382.

14 Hidalgo A, Franquet T, Gimenez A, et al. Smoking-related interstitial lung diseases: radiologic-pathologic correlation. Eur Radiol 2006; 16: 2463-2470.

15 Godbert B, Wissler MP, Vignaud JM. Desquamative interstitial pneumonia: an analytic review with an emphasis on aetiology. Eur Respir Rev 2013; 22: 117-123.

16 Hellemons ME, Moor CC, von der Thüsen J, et al. Desquamative interstitial pneumonia: a systematic review of its features and outcomes. Eur Respir Rev 2020; 29: 190181.

17 Carrington CB, Gaensler EA, Coutu RE, et al. Natural history and treated course of usual and desquamative interstitial pneumonia. N Engl J Med 1978; 298: 801-809.

18 Nicholson AG, Colby TV, du Bois RM, et al. The prognostic significance of the histologic pattern of interstitial pneumonia in patients presenting with the clinical entity of cryptogenic fibrosing alveolitis. Am J Respir Crit Care Med 2000; 162: 2213-2217. 
19 Winterbauer RH, Hammar SP, Hallman KO, et al. Diffuse interstitial pneumonitis. Clinicopathologic correlations in 20 patients treated with prednisone/azathioprine. Am J Med 1978; 65: 661-672.

20 Kawabata Y, Takemura T, Hebisawa A, et al. Desquamative interstitial pneumonia may progress to lung fibrosis as characterized radiologically. Respirology 2012; 17: 1214-1221.

21 Ryu JH, Myers JL, Capizzi SA, et al. Desquamative interstitial pneumonia and respiratory bronchiolitis-associated interstitial lung disease. Chest 2005; 127: 178-184.

22 Akira M, Yamamoto S, Hara H, et al. Serial computed tomographic evaluation in desquamative interstitial pneumonia. Thorax 1997; 52: 333-337.

23 Knyazhitskiy A, Masson RG, Corkey R, et al. Beneficial response to macrolide antibiotic in a patient with desquamative interstitial pneumonia refractory to corticosteroid therapy. Chest 2008; 134: 185-187.

24 Flaherty KR, Wells AU, Cottin V, et al. Nintedanib in progressive fibrosing interstitial lung diseases. $N$ Engl J Med 2019; 381: 1718-1727.

25 King MB, Jessurun J, Hertz MI. Recurrence of desquamative interstitial pneumonia after lung transplantation. Am J Respir Crit Care Med 1997; 156: 2003-2005.

26 Portnoy J, Veraldi KL, Schwarz MI, et al. Respiratory bronchiolitis-interstitial lung disease: long-term outcome. Chest 2007; 131: 664-671.

27 Nakanishi M, Demura Y, Mizuno S, et al. Changes in HRCT findings in patients with respiratory bronchiolitis-associated interstitial lung disease after smoking cessation. Eur Respir J 2007; 29: 453-461. 